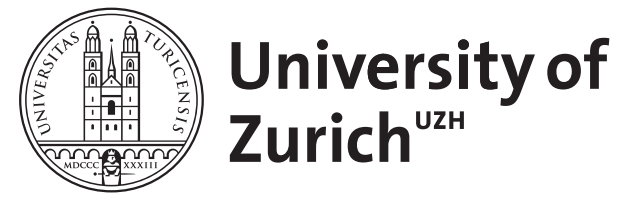

\title{
Subgingival biofilm structure
}

\author{
Zijnge, Vincent ; Ammann, Thomas ; Thurnheer, Thomas ; Gmür, Rudolf
}

\begin{abstract}
Periodontitis is an inflammatory disease of the oral cavity initiated by a microbial biofilm (or 'dental plaque'). Subgingival biofilms in periodontal pockets are not easily analyzed without the loss of structural integrity. These subgingival plaques are structured communities of microorganisms with great phylogenetic diversity embedded in a self-produced extracellular polymeric matrix. For almost three decades, knowledge of the structure of plaque located below the gingival margin has been limited to landmark studies from the 1970s that were unaware of the breadth of microbial diversity we appreciate now. Only recently has technical progress - combining histology, confocal scanning fluorescent microscopy and fluorescent in situ hybridization to localize the most abundant species from different phyla and species associated with periodontitis - provided new insights into the architecture of subgingival biofilms. This review focuses on the structure and composition of subgingival biofilms and discusses current knowledge on the nature of the extracellular matrix. We describe further structural aspects of 'subgingival' biofilms produced in vitro that are gaining considerable interest as we search for models to investigate biofilm development, resistance to antibiotics, extracellular polymeric matrix composition and function, and reciprocal host-cell-to-biofilm interactions.
\end{abstract}

DOI: https://doi.org/10.1159/000329667

Posted at the Zurich Open Repository and Archive, University of Zurich ZORA URL: https://doi.org/10.5167/uzh-64502

Journal Article

Accepted Version

Originally published at:

Zijnge, Vincent; Ammann, Thomas; Thurnheer, Thomas; Gmür, Rudolf (2012). Subgingival biofilm structure. Frontiers of Oral Biology, 15:1-16.

DOI: https://doi.org/10.1159/000329667 


\section{Subgingival biofilm structure}

Vincent Zijnge ${ }^{1}$, Thomas Ammann², Thomas Thurnheer², Rudolf Gmür²

${ }^{1}$ Dept. of Oral Microbiology University of Umeå, Umeå, Sweden, and ${ }^{2}$ Institute of Oral

Biology, Section of Oral Microbiology and General Immunology, University of Zürich, Zürich, Switzerland

Running title: Subgingival biofilm structure

${ }^{*}$ Corresponding author. Mailing address: ${ }^{1}$ Institute of Oral Biology, Section of Oral Microbiology and General Immunology, University of Zürich, Plattenstrasse 11, CH-8032

Zürich, Switzerland. Phone: +41-44-6343306. Fax: +41-44-6344310. E-mail:

rudolf.gmuer@zzm.uzh.ch 


\begin{abstract}
Periodontitis is an inflammatory disease of the oral cavity initiated by a microbial biofilm ("or dental plaque"). Subgingival biofilms in periodontal pockets are not easily analyzed without the loss of structural integrity. These subgingival plaques are structured communities of microorganisms with great phylogenetic diversity embedded in a self-produced extracellular polymeric matrix (EPM). For almost three decades knowledge on the structure of plaque located below the gingival margin has been limited to landmark studies from the 1970 s that were unaware of the breadth of microbial diversity we appreciate now. Only recently, technical progress combining histology, confocal scanning fluorescent microscopy and fluorescent in situ hybridization to localize the most abundant species from different phyla and species associated with periodontitis, provided new insights into the architecture of subgingival biofilms. This review focuses on the structure and composition of subgingival biofilms and discusses current knowledge on the nature of the extracellular matrix. We describe further structural aspects of in vitro produced 'subgingival' biofilms that are gaining considerable interest as we search for models to investigate biofilm development, resistance to antibiotics, EPM composition and function, and reciprocal host-cell to biofilm interactions.
\end{abstract}




\section{General introduction}

Microbial dental plaque, regardless of differences in location and composition, consist of adherent consortia of microorganisms (mostly bacteria, few archaea, viruses, yeasts, amoebae, and a virtually unknown, presumably large, population of bacteriophages) that fulfill the consensus definition of biofilms. This definition describes "... a bacterial biofilm as a structured community of bacterial cells enclosed in a self-produced (hydrated) polymeric matrix and adherent to an inert or living surface" [1]. Subgingival biofilms are threedimensional (3-D), structured communities of bacteria that live attached to the surface of the root of teeth or dental implants, with their outer surface directly facing the gingival tissue. In a healthy periodontium these sites are not accessible to bacteria. But persistence of biofilm at the gingival margin and in the gingival sulcus leads to gingivitis, a reversible condition, which in susceptible patients may progress to periodontitis characterized by the irreversible loss of the tooth-supporting structures.

A key-feature of this destructive inflammatory process is the formation of deep gingival pockets, which are colonized by the biofilm as they develop. The biofilm bacteria are embedded in a poorly understood extracellular matrix composed of exo-polysaccharides, proteins and extracellular DNA. The formation of subgingival biofilm and its continuous adaptation to changing environmental conditions is governed by a dynamic equilibrium between the microorganisms, the cellular and humoral host defense, and a multitude of anabolic and catabolic products and signaling factors produced by both the microbiota and the periodontal tissues [2]. This results in a complex biofilm ecology where bacteria behave fundamentally different than in suspension cultures where they exhibit a planktonic existence.

Max A. Listgarten, a pioneer of structural analyses of oral biofilms, while still at UPenn in 1994, published an excellent review on 'the structure of dental plaque' [2], which summarized the relevant work done up to that time point and highlighted the concepts on the relationship 
of biofilm structure to clinical status and the clinical relevance of biofilm composition and structure. The review was written at a time when new molecular microbiological techniques began to conquer the dental field [3]. Eventually, this new development lead to an explosion of information on the complexity and diversity of biofilm composition not imaginable at that time. It is the intent of this chapter to build on Listgarten's review [2] and update our current understanding of subgingival biofilms in light of the progress that the technical and conceptual developments of the last 15 years have brought with regard to biofilm diversity, in vivo and in vitro biofilm architecture and extracellular matrix composition.

\section{Improved analytical procedures reveal complex subgingival biofilm composition}

Extensive culture analyses performed in the 1980 s and early 1990 s to determine the predominant cultivable subgingival microbiota showed that this biofilm may harbor as much as $10^{9}$ bacteria and more than 100 different species in a single pocket $[4,5]$. If the microbiota of entire study cohorts rather than individual pockets are considered, much larger diversity is apparent and suggests that on a population basis, more than 500 bacterial species might be found in samples from the human oral cavity [4]. Some subgingival species, such as Aggregatibacter actinomycetemcomitans, Porphyromonas gingivalis, and Tannerella forsythia, stood out in that they were found with increased prevalence and in clearly elevated numbers at diseased sites in comparison to healthy control sites. In 1996, these species were designated as periodontal pathogens in the Consensus Report of the World Workshop in Periodontology. Today they figure among the best studied medically important bacteria and in many respects have become model organisms. Nevertheless, it was clear all the time that other cultivable and non-cultivable subgingival species may be equally important and would merit further investigation [5]. Spirochetes, accounting for as much as $50 \%$ of the microscopically detectable subgingival microbiota, may serve as a prime example. But do we need to describe the microbial community structure in a taxonomical precise and quantitative way? Amann and Ludwig [6] raised the question in a review 10 years ago and answered it with: "Yes, of course!" Why? Because complex microbial ecologies cannot be 
characterized sufficiently by selected individual taxa that may have particularly well adapted to their ecological niche. Complex microbial ecologies are rather defined by all the mutual interactions that determine the abundance, the localization and the activities of their members [6]. Clearly, new tools for studies of (oral) microbial diversity were required. These became available in the mid-1980s with the emergence of new procedures for the comparative sequencing of homologous biopolymers [7]. In particular the extensive sequencing of ribosomal RNA (16S and to a lesser extent 23S rRNA) became very attractive and influential as mirrored by the now more than $1.4 \times 10^{6}$ deposited $16 \mathrm{~S}$ rRNA sequences (Ribosomal Database Project August 2010; http://rdp.cme.msu.edu). Paster and co-workers described in 2001215 novel phylotypes associated with subgingival plaque [8]. In the following years, numerous conceptually and technically similar studies complemented each other and lengthened the list of newly recognized species and phylotypes, culminating in the description and most recently in an update of the Human Oral Microbiome (HOMD) database (http://www.homd.org) [9]. This database includes a backbone-library of about 800 16S rRNA gene sequences that are structured phylogenetically and taxonomically into 619 taxa in 13 phyla. The backbone-library will be expanded in the near future by data from an additional 36 '000 clonal sequences of which approximately $10 \%$ - accounting for as much as 654 new taxa - are not yet covered by the current HOMD backbone-database [9]. In addition to this, recent progress in DNA sequencing technology ("pyrosequencing") has brought an enormous increase of nucleotide sequences of oral microbial origin, resulting in an overwhelming number of phylotypes of unknown species affiliation [10-12]. Where in the biofilms are the taxa located? How abundant are they? What are their morphologies, their physiological activities, their importance for both biofilm and host-biofilm relationship? To a large part we do not know - yet. Recently, technical progress has provided very promising possibilities for the single-cell identification and localization in intact natural subgingival biofilms of most taxa and phylogentic clusters with an available 16S rRNA sequence.

\section{Methods to study subgingival biofilm architecture}


Some of the most valuable information on supragingival biofilm formation, ecology and architecture has come from in situ studies performed with volunteers wearing expoxy crowns [13], artificial surfaces attached to selected teeth [14], or specifically designed appliances into which enamel, dentin, glass, or plastic slabs had been inserted [e.g. 15-17]. While all these studies could only identify the biofilm bacteria based on morphology or cell wall structure (if electron microscopy was used) and differentiate between live and dead organisms using vitality stains, some more recent studies used fluorescent in situ hybridization (FISH) technologies to identify targeted biofilm bacteria at the species level [e.g. 18-21]. The application of phylogenetic group- or species-specific single cell identification techniques to undisturbed biofilms formed supragingivally in the oral cavity on retrievable surfaces is currently the state of the art and promises to reveal important new information on supragingival plaque formation and architecture.

Unfortunately, the study of subgingival plaque is much more difficult in comparison to supragingival plaque due the protected location of periodontal pockets. Accordingly, the development of the three-dimensional subgingival biofilm structure is less well characterized. Access to undisturbed natural subgingival biofilms can only be gained by tooth extraction. Listgarten and co-workers pioneered structural analyses of oral biofilms more than three decades ago using light and electron microscopy [22]. The major hindrance of their groundbreaking studies was the impossibility at the time to identify the detected bacteria beyond cell morphology (cell shape, cell wall structure, and Gram-stain). This obstacle has been overcome only recently when entire teeth affected by advanced periodontitis were extracted without disturbance of the adherent subgingival biofilm and, after immediate fixation and processing to serial sections of $2 \mu \mathrm{m}$ thickness, were stained by FISH using various combinations of group- and species-specific rRNA probes and studied by epifluorescence or confocal laser scanning microscopy (CLSM) [23]. A way to investigate in situ subgingival biofilm formation without the loss of the tooth under study was developed by Wecke et al. [24] and over the last few years refined and applied in several studies [25-27]. This procedure uses gold foil or small plastic carriers covered with expanded 
polytetrafluoroethylene membranes, which are inserted into the depth of periodontal pockets for defined periods of time and then processed similarly to extracted teeth. In the following, these recently published studies are reviewed in greater detail as they may lead the track to further investigations that likely will be carried out using much broader selections of phylogenetic group-, genus-, and species-specific FISH probes to elucidate the subgingival biofilm architecture.

\section{Subgingival plaque structure}

A profound understanding of the in vivo structure of subgingival biofilms, i.e. the natural situation, is essential for designing and interpreting diversity measurements and in vitro experimental biofilms. Histological slices perpendicular to the root surface reveal three easily envisioned and clinical relevant areas, viz. the bottom of the pocket, the root surface and the epithelial side [28]. At the bottom of the pocket the periodontal ligament and the gingival connective tissue border the biofilm. This is the area where further attachment loss occurs in progressing periodontitis and where further pristine tooth surface becomes colonized. The species or bacterial cell morphologies observed in this area are filamentous, large rodshaped, spirochete-shaped and branching. Especially the branching cells are abundant, whereas spirochetes are sometimes missing [29].

The bulk of the biofilm mass is situated along the root surface with histologically defined parts adhering to the root surface and facing the gingival host tissue. Listgarten was the first to describe the structure of such subgingival biofilms in 1976 using light and electron microscopy [22]. His pioneering work with natural teeth affected by periodontal disease provided the first informative glimpses at subgingival plaque structure. The pictures of subgingival biofilms showed a wealth of different cell morphologies like cocci, rods, fusiforms, spirochetes, flagellated bacteria, small and very large forms, and bacterial aggregates. Moreover, the biofilm itself showed a distinct organization, indicating that biofilms might be structured entities themselves that are beneficial to their inhabitants. 
The images shown in Fig. 1 show a remarkable and unprecedented agreement between samples taken at a time interval of 30 years and analyzed with different techniques $[22,23]$. They provide a clear overview of the different structures that can be distinguished when analyzing subgingival biofilms. Three different zones are observed between the root surface and the gingival tissue lining of the periodontal pocket. In the zone directly adhering to the root surface, relatively small cells without any particular orientation are visible (Figs. 1, 2). These bacteria are embedded in somewhat fibrous, membrane-like material that may consist of debris, dead cells and biofilm matrix components. Amongst these unidentified bacteria with various morphologies, other bacteria can be recognized as Actinobacteria due to their characteristic branching or labeling by FISH (Fig. 2) [23]. On top of that layer a structurally different layer of bacteria can be observed that is more compact and electron dense (Figs. 1, 2). This typical intermediate layer contains distinctive bacteria that are filamentous or rod shaped and is without a well-defined intercellular matrix [22, 30]. FISH has identified these bacteria in part as Fusobacterium nucleatum and T. forsythia (Fig. 3) [23]. The outermost part of the adhering biofilm that is facing the host tissues is characterized by a palisading layer of so-called "test tube brushes" and of large parallel cells that are orientated with their long axis perpendicular to the root surface (Figs. 1, 2). The test tube brushes consist of one or several large central filaments surrounded by gram-negative rods or small filaments. The parallel-orientated cells have been identified as members of the Synergistetes phylum (Fig. 4) $[23,31]$ and represent a lineage composed of taxa that for the most part are not yet cultivable. Opposing the Synergistetes in this outermost part of the adhering biofilm, polymorphonuclear neutrophils (PMNs) can be observed by light, electron and epifluorescent microscopy (Figs. 1, 2, 4, 6) [22, 28]. Several reports described an additional, loose layer of bacteria covering the adhering biofilm without a clear cellular organization and dominated by flagellated and spirochetal cells belonging to Treponema and Selenomonas species [23, 26, 32] although rods, filaments, cocci and test tube brushes can also be observed (Fig. 5). A clear demarcation between the host tissue and this loose bacterial layer is not recognizable, 
probably because severe inflammation induces immunopathological tissue damage accompanied by collagen loss, ulceration, and marked tissue permeabilization.

The subgingival biofilm shows a stratification from the root surface towards the epithelium lining. The basal, intermediate, top and outer layers seem to provide a general structure of the biofilm with corresponding species associated with each layer. Actinobacteria sp., $F$. nucleatum, T. forsythia, Synergistetes sp. and Treponema sp. appear to make up a framework of the subgingival biofilm. Observations with species-specific probes and goldlabeled antibodies show further species that colonize within this biofilm framework by forming distinct microcolonies. In the outer layer of the adhering biofilm, closest to the epithelium and cells of the host defense, aggregates or microcolonies of Prevotella intermedia, Porphyromonas endodontalis, P. gingivalis, and Parvimonas micra (formerly Peptostreptococcus micros) have been observed (Fig. 6). Microbial biofilms are dynamic communities exposed to an ever-changing environment. Therefore, the outlined architecture of the subgingival biofilm should not be seen as a rigid structure but rather as a continuously changing consortium that is influenced by bacterial growth, attachment and detachment, the host's inflammatory immune response, the host's oral hygiene measures, and the nutritional conditions defined by the ecological niche. The availability or lack of nutrients is of course directly linked to both biofilm composition and extent of inflammation. Although the general setup of the subgingival biofilm framework appears relatively uniform, less common species may be observed in diverse sites, in particular within the loose layer that covers the adhering biofilm.

Clearly, our understanding of the subgingival plaque structure is incomplete. The number of teeth studied, the number of patients who provided teeth, and the number and specificities of the employed FISH probes are too limited for more precise conclusions. The degree of diversity currently appreciated among plaque organisms [9] is not reflected in the currently available studies of biofilm architecture. The application of rRNA FISH probes with specificities for large phylogenetic groups such as $\beta$-, $\gamma$ - or $\varepsilon$-Proteobacteria, Bacteroidetes, 
Eubacteria sp., Selenomonas sp. etc. to subgingival plaque samples that had been dispersed and subjected to FISH on multi-well glass slides, indicates that other bacterial taxa may account for substantial amounts of the biofilm (Gmür et al., unpublished data).

\section{Structure of in vitro formed "subgingival" biofilms}

It is evident that these natural subgingival biofilms are extremely complex and sited in a barely accessible, fluctuating, inflammation-affected environment. Together with ethical restrictions this limits in vivo experimentation and makes interpretation of results difficult. Several research groups have developed in vitro model systems of subgingival plaque with the aim to reduce complexity while maintaining the bacterial biofilm "lifestyle" and reproducing characteristic properties of such communities [e.g. 33-36]. Regardless whether freshly collected, dispersed human subgingival plaque or balanced mixtures of strains of subgingival species were used to initiate biofilm formation, the nutritional conditions were recognized to have a dominant influence on biofilm development [35, 36]. This must have profound effects on biofilm structure, but information on the architecture of such in vitro generated subgingival biofilms is still scarce $[34,36]$. Figure 7 shows images from an own ongoing study (Ammann et al., unpublished) investigating the architecture of 10-species 'subgingival' biofilms grown in vitro in a saliva-free standard fluid medium supplemented with human serum (50\%:50\%). The biofilms reached a thickness of $70-100 \mu \mathrm{m}$ after $64.5 \mathrm{~h}$ of incubation with all inoculated species at detectable levels. F. nucleatum, Streptococcus oralis and Streptococcus anginosus predominated and were found throughout the biofilm (Fig. 7 D). Streptococci did not grow dispersed but formed large compact aggregates, often surrounded by layers of Veillonella dispar (Fig. 7 D). A. naes/undii was detected in relatively large individual colonies (Fig. 7 A, B) embedded in a readily detectable extracellular polysaccharide matrix (not shown) and spreading from the bottom third to the top of the biofilm. This suggests that $A$. naeslundii expanded rapidly from a relatively limited number of foci and, with a little longer incubation period, would have possibly spread over the entire body of the biofilm. This would correspond to the spatial distribution seen with in vivo biofilms 
(Fig. 2). In contrast, Treponema denticola and P. gingivalis were detected selectively in close proximity to each other atop of very dense brightly stained cushions of bacteria (presumably streptococci) at the biofilm surface (Fig. 7 C). The presence of spirochetes and Porphyromonas species at this location is a characteristic attribute of subgingival plaque (Figs. 5,6), hence it will be important to further analyze in vitro the reproducibility of this interesting finding. It must be emphasized that these are preliminary data gained with a limited number of biofilms. However, they demonstrate that the combination of 3-D CLSM and multiplex FISH promises to be of great value in further elucidating the architecture of both natural and artificially generated subgingival biofilms.

\section{Extracellular matrix composition}

There are as many different types of biofilms as there are bacteria [37]. But in any biofilm a substantial part consists of material other than bacteria. In general it is estimated that microorganisms account for less than $10 \%$ of the dry weight of biofilms and that the extracellular matrix contributes over $90 \%$ [38]. The formation of the extracellular matrix is generally recognized as the second stage during biofilm formation, after initial adherence and proliferation of microorganisms [39]. The extracellular matrix is composed of a heterogeneous mixture of polysaccharides, proteins and extracellular DNA (eDNA), which are called collectively extracellular polymeric substances (EPS). These complex conglomerate EPS are very difficult to analyze. Hence it is not surprising that current knowledge on biofilm associated EPS is derived almost exclusively from single-species biofilm models. However, whether the EPS production by these bacteria is the same when they live within a complex "biofilm-city" [40] remains to be seen.

Extracellular polysaccharides occur in two basic forms. They can be associated with the cell surface and form a capsule or be secreted as a slimy biofilm matrix. Most polysaccharides are long linear or branched molecules composed of multiple saccharide units like glucose, fructose or sucrose and exist as homo- or heteropolysaccharides. Many of them possess acyl-groups and organic and inorganic substituents like acetate, pyruvate or sulphate. These 
substituents and the presence of charged sugar residues largely determine the physical properties of the extracellular polysaccharides. The type of polysaccharide that is produced usually varies among the different species and some species can produce multiple kinds of polysaccharides [37]. One of the most common studied matrix polysaccharides is beta-1,6-Nacetyl-D-glucosamine called PNAG or PGA. It is produced, among others, by $A$. actinomycetemcomitans for biofilm formation. In A. actinomycetemcomitans PGA mediates intercellular adhesion and contributes to biofilm cohesion, but also protects the cells against killing by macrophages [41]. Prevotella nigrescens produces a heteropolysaccharide that is composed mainly of mannose, but also contains other sugars, including glucose and fructose [42]. A mannose rich polysaccharide has also been identified from $P$. intermedia $[43,44]$. The polysaccharides produced by $P$. nigrescens and $P$. intermedia contribute to biofilm formation and the resistance to neutrophil phagocytosis. $P$. gingivalis strains on the other hand produce a capsular polysaccharide. Encapsulated strains have been shown to evade the immune system [45] but non-encapsulated strains are more adherent to epithelial cells and show strong autoaggregation and enhanced biofilm formation $[46,47]$. If and what type of free extracellular polysaccharide $P$. gingivalis produces for the formation of biofilms is unknown. Actinobacteria produce levan which is a homopolysaccharide composed of beta $(2 \rightarrow 6)$ linked fructosyl units [48]. Levan may function as extracellular storage polymers, but has also been shown to stimulate the inflammatory response. The contribution of levans to Actinobacteria biofilm formation is unknown.

Extracellular proteins that can be found in, and contribute to, a biofilm are lectins and sugar binding proteins that facilitate cell-to-cell or cell-to-matrix interactions [49]. A second group of extracellular proteins involved in cell-to cell or cell-to-matrix interactions are autotransporters. Autotransporters transport themselves across the outer membrane of gram-negative bacteria and may function in adherence and biofilm formation. A third group of extracellular proteins that structurally contribute to the biofilm matrix are pili, also called fimbriae [37]. A type I and a type IV pilus are distinguished. The type I pilus consist of a long rigid structure of repeating subunits that is attached to the cell with a fimbrial tip that recognizes specific substrates. 
Type I pili have been observed on a number of oral bacteria [50] including $P$. gingivalis, $A$. naeslundii [51] and P. intermedia [52]. In Actinomyces oris type I pili are functional in biofilm formation [53]. Type IV pili are often located at one of the poles of the cell and may provide the cell with twitching motility. The pilus is a fiber composed of repeating units of pilin encoded by the PilA gene [54]. Type IV pili have been identified in Eikenella corrodens and A. actinomycetemcomitans $[50,55]$. Genomic screening indicated the presence of type IV pili as in F. nucleatum [54]. In A. actinomycetemcomitans the type IV pilus is considered to be a distinct subtype assembled as bundles of long, thin fibrils encoded by the tad locus [55]. $A$. actinomycetemcomitans strains deficient in the formation of pili form relatively fragile biofilms $[56]$.

Extracellular DNA has been shown to be an important constituent of biofilms and to contribute to biofilm integrity $[38,57,58]$. There are indications that eDNA results from the controlled lysis of cells as well as from the active release of DNA containing membrane vesicles by viable cells $[37,38]$. It is tempting to speculate that eDNA might function as a grid along which bacteria can move through the biofilm by using Type IV pili with DNA-specific binding sites. There is no doubt that the characteristics of the extracellular polymeric substances contribute to the specific properties of the biofilm. Many components are hygroscopic and keep the biofilm hydrated and prevent the cells from drying. The EPS matrix can act as a molecular sieve and protect the bacteria in the biofilm from toxic compounds or host immune responses and nutrients are accumulated. The enhanced resistance of biofilms in comparison to planktonic bacteria to antimicrobial agents is well established [59]. The entanglement of biopolymers provides the biofilm with the physical strength and flexibility to withstand mechanical forces and shear stress. While biofilms provide an optimal environment for bacterial growth and survival, external impacts that may cause starvation or altered bacterial cross talk are known to induce the production of enzymes that can potentially degrade EPS components. For example, A. actinomycetemcomitans produces the extracellular $N$-acetyl- $\beta$-hexoseaminidase dispersin B that hydrolyzes the glycosidic links in PNAG and degrades the biofilm matrix. This may result in the dispersal of cells or clumps of 
cells, which in turn can colonize new surfaces and initiate a new biofilm lifecycle $[38,57]$.

Flemming and Wingender concluded in their recent review that "despite much research on biofilms, basic questions remain" and continued that "a better understanding of the regulation of EPS production in mixed-species biofilms, as well as a spatial and temporal dissection of the phases in EPS production, will reveal important aspects of the oldest, most successful and widespread form of life on Earth" [38].

\section{Acknowledgments}

TA is supported by the research fund of the Swiss Dental Association.

\section{References}

1 Costerton JW, Stewart PS, Greenberg EP: Bacterial biofilms: A common cause of persistent infections. Science 1999;284:1318-1322.

2 Listgarten MA: The structure of dental plaque. Periodontol 2000 1994;5:52-65.

3 Tanner A, Maiden MFJ, Paster BJ, Dewhirst FE: The impact of $16 \mathrm{~S}$ ribosomal RNAbased phylogeny on the taxonomy of oral bacteria. Periodontol 2000 1994;5:26-51.

4 Moore WEC, Moore LVH: The bacteria of periodontal diseases. Periodontol 2000 1994;5:66-77.

$5 \quad$ Haffajee AD, Socransky SS: Micobial etiological agents of destructive periodontal diseases. Periodontol 2000 1994;5:78-111.

6 Amann R, Ludwig W: Ribosomal RNA-targeted nucleic acid probes for studies in microbial ecology. FEMS Microbiol Rev 2000;24:555-565.

7 Amann RI, Binder BJ, Olson RJ, Chisholm SW, Devereux R, Stahl DA: Combination of 16S rRNA-targeted oligonucleotide probes with flow cytometry for analyzing mixed microbial populations. Appl Environ Microbiol 1990;56:1919-1925.

8 Paster BJ, Boches SK, Galvin JL, Ericson RE, Lau CN, Levanos VA, Sahasrabudhe A, Dewhirst FE: Bacterial diversity in human subgingival plaque. J Bacteriol 2001;183:3770-3783. 
9 Dewhirst FE, Chen T, Izard J, Paster BJ, Tanner AC, Yu WH, Lakshmanan A, Wade WG: The human oral microbiome. J Bacteriol 2010;192:5002-5017.

10 Keijser BJ, Zaura E, Huse SM, van der Vossen JM, Schuren FH, Montijn RC, ten Cate $\mathrm{JM}$, Crielaard W: Pyrosequencing analysis of the oral microflora of healthy adults. $\mathrm{J}$ Dent Res 2008;87:1016-1020.

11 Zaura E, Keijser BJF, Huse SM, Crielaard W: Defining the healthy "core microbiome" of oral microbial communities. BMC Microbiol 2009;9:259.

12 Contreras M, Costello EK, Hidalgo G, Magris M, Knight R, Dominguez-Bello MG: The bacterial microbiota in the oral mucosa of rural Amerindians. Microbiology 2010;156:3282-3287.

13 Listgarten M, Mayo HE, Tremblay R: Development of dental plaque on epoxy resin crowns in man. J Periodontol 1975;46:10-26.

14 Wood SR, Kirkham J, Marsh PD, Shore RC, Nattress B, Robinson C: Architecture of intact natural human plaque biofilms studied by confocal laser scanning microscopy. $\mathrm{J}$ Dent Res 2000;79:21-27.

15 Netuschil L, Reich E, Unteregger G, Sculean A, Brecx M: A pilot study of confocal laser scanning microscopy for the assessment of undisturbed dental plaque vitality and topography. Arch Oral Biol 1998;43:277-285.

16 Zaura-Arite E, van Marle J, ten Cate JM: Confocal microscopy study of undisturbed and chlorhexidine-treated dental biofilm. J Dent Res 2001;80:1436-1440.

17 Wood SR, Kirkham J, Shore RC, Brookes SJ, Robinson C: Changes in the structure and density of oral plaque biofilms with increasing plaque age. FEMS Microbiol Ecol 2002;39:239-244

18 Foster JS, Palmer RJJ, Kolenbrander PE: Human oral cavity as a model for the study of genome-genome interactions. Biol Bull 2003;204:200-204.

19 Palmer RJ, Jr., Diaz PI, Kolenbrander PE: Rapid succession within the Veillonella population of a developing human oral biofilm in situ. J Bacteriol 2006;188:4117-4124. 
20 Al-Ahmad A, Wunder A, Auschill TM, Follo M, Braun G, Hellwig E, Arweiler NB: The in vivo dynamics of Streptococcus spp., Actinomyces naes/undii, Fusobacterium nucleatum and Veillonella spp. in dental plaque biofilm as analysed by five-colour multiplex fluorescence in situ hybridization. J Med Microbiol 2007;56:681-687.

21 Dige I, Raarup MK, Nyengaard JR, Kilian M, Nyvad B: Actinomyces naeslundii in initial dental biofilm formation. Microbiology 2009;155:2116-2126.

22 Listgarten MA: Structure of the microbial flora associated with periodontal health and disease in man. A light and electron microscopic study. J Periodontol 1976;47:1-18.

23 Zijnge V, van Leeuwen MBM, Degener JE, Abbas F, Thurnheer T, Gmür R, Harmsen HJM: Oral biofilm architecture on natural teeth. PLoS ONE 2010;5:e9321.

24 Wecke J, Kersten T, Madela K, Moter A, Göbel UB, Friedmann A, Bernimoulin J-P: A novel technique for monitoring the development of bacterial biofilms in human periodontal pockets. FEMS Microbiol Lett 2000;191:95-101.

25 Moter A, Göbel UB: Fluorescence in situ hybridization (FISH) for direct visualization of microorganisms. J Microbiol Methods 2000;41:85-112.

26 Drescher J, Schlafer S, Schaudinn C, Riep B, Neumann K, Friedmann A, Petrich A, Göbel UB, Moter A: Molecular epidemiology and spatial distribution of Selenomonas spp. in subgingival biofilms. Eur J Oral Sci 2010;118:466-474.

27 Schlafer S, Riep B, Griffen AL, Petrich A, Hübner J, Berning M, Friedmann A, Göbel UB, Moter A: Filifactor alocis - involvement in periodontal biofilms. BMC Microbiol 2010;10:66.

28 Waerhaug J: Subgingival plaque and loss of attachment in periodontosis as observed in autopsy material. J Periodontol 1976;47:636-642.

29 Saglie R: A scanning electron microscopic study of the relationship between the most apically located subgingival plaque and the epithelial attachment. J Periodontol 1977;48:105-115.

30 Westergaard J, Frandsen A, Slots J: Ultrastructure of the subgingival microflora in juvenile periodontitis. Scand J Dent Res 1978;86:421-429. 
31 Vartoukian SR, Palmer RM, Wade WG: Diversity and morphology of members of the phylum "Synergistetes" in periodontal health and disease. Appl Environ Microbiol 2009;75:3777-3786.

32 Noiri Y, Li L, Ebisu S: The localization of periodontal-disease-associated bacteria in human periodontal pockets. J Dent Res 2001;80:1930-1934.

33 Bradshaw DJ, Marsh PD, Watson GK, Allison C: Role of Fusobacterium nucleatum and coaggregation in anaerobe survival in planctonic and biofilm oral microbial communities during aeration. Infect Immun 1998;66:4729-4732.

34 Hope CK, Wilson M: Biofilm structure and cell vitality in a laboratory model of subgingival plaque. J Microbiol Methods 2006;66:390-398.

35 Walker C, Sedlacek MJ: An in vitro biofilm model of subgingival plaque. Oral Microbiol Immunol 2007;22:152-161.

36 Guggenheim B, Gmür R, Galicia JC, Stathopoulou P, Benakanakere MR, Meier A, Thurnheer T, Kinane D: In vitro modeling of host-parasite interactions: the 'subgingival' biofilm challenge of primary human epithelial cells. BMC Microbiol 2009;9:280.

37 Karatan E, Watnick P: Signals, regulatory networks, and materials that build and break bacterial biofilms. Microbiol Mol Biol Rev 2009;73:310-347.

38 Flemming HC, Wingender J: The biofilm matrix. Nat Rev Microbiol 2010;8:623-633.

39 O'Toole G, Kaplan HB, Kolter R: Biofilm formation as microbial development. Annu Rev Microbiol 2000;54:49-79.

40 Watnick P, Kolter R: Biofilm, city of microbes. J Bacteriol 2001;182:2675-2679.

41 Venketaraman V, Lin AK, Le A, Kachlany SC, Connell ND, Kaplan JB: Both leukotoxin and poly- $N$-acetylglucosamine surface polysaccharide protect Aggregatibacter actinomycetemcomitans cells from macrophage killing. Microb Pathog 2008;45:173180.

42 Yamane K, Yamanaka T, Yamamoto N, Furukawa T, Fukushima H, Walker CB, Leung KP: A novel exopolysaccharide from a clinical isolate of Prevotella nigrescens: 
purification, chemical characterization and possible role in modifying human leukocyte phagocytosis. Oral Microbiol Immunol 2005;20:1-9.

43 Fukushima H, Moroi H, Inoue J, Onoe T, Ezaki T, Yabuuchi E, Leung K-P, Walker CB, Clark WB, Sagawa H: Phenotypic characteristics and DNA relatedness in Prevotella intermedia and similar organisms. Oral Microbiol Immunol 1992;7:60-64.

44 Yamanaka T, Furukawa T, Matsumoto-Mashimo C, Yamane K, Sugimori C, Nambu T, Mori N, Nishikawa H, Walker CB, Leung KP, Fukushima H: Gene expression profile and pathogenicity of biofilm-forming Prevotella intermedia strain 17. BMC Microbiol 2009;9:11.

45 Brunner J, Scheres N, El Idrissi NB, Deng DM, Laine ML, van Winkelhoff AJ, Crielaard W: The capsule of Porphyromonas gingivalis reduces the immune response of human gingival fibroblasts. BMC Microbiol 2010;10:5.

46 Davey ME, Duncan MJ: Enhanced biofilm formation and loss of capsule synthesis: deletion of a putative glycosyltransferase in Porphyromonas gingivalis. J Bacteriol 2006;188:5510-5523.

47 Yamaguchi M, Sato K, Yukitake H, Noiri Y, Ebisu S, Nakayama K: A Porphyromonas gingivalis mutant defective in a putative glycosyltransferase exhibits defective biosynthesis of the polysaccharide portions of lipopolysaccharide, decreased gingipain activities, strong autoaggregation, and increased biofilm formation. Infect Immun 2010;78:3801-3812.

48 Yeung MK: Molecular and genetic analyses of Actinomyces spp. Crit Rev Oral Biol Med 1999;10:120-138.

49 Whittaker CJ, Klier CM, Kolenbrander PE: Mechanisms of adhesion by oral bacteria. Annu Rev Microbiol 1996;50:513-552.

$50 \mathrm{Wu}$ H, Fives-Taylor PM: Molecular strategies for fimbrial expression and assembly. Crit Rev Oral Biol Med 2001;12:101-115. 
51 Chen P, Cisar JO, Hess S, Ho JT, Leung KP: Amended description of the genes for synthesis of Actinomyces naeslundii T14V type 1 fimbriae and associated adhesin. Infect Immun 2007;75:4181-4185.

52 Dorn BR, Leung K-P, Progulske-Fox A: Invasion of human oral epithelial cells by Prevotella intermedia. Infect Immun 1998;66:6054-6057.

53 Mishra A, Wu C, Yang J, Cisar JO, Das A, Ton-That H: The Actinomyces oris type 2 fimbrial shaft FimA mediates co-aggregation with oral streptococci, adherence to red blood cells and biofilm development. Mol Microbiol 2010;77:841-854.

54 Desvaux M, Khan A, Beatson SA, Scott-Tucker A, Henderson IR: Protein secretion systems in Fusobacterium nucleatum: genomic identification of Type 4 piliation and complete Type $\mathrm{V}$ pathways brings new insight into mechanisms of pathogenesis. Biochim Biophys Acta 2005;1713:92-112.

55 Tomich M, Planet PJ, Figurski DH: The tad locus: postcards from the widespread colonization island. Nat Rev Microbiol 2007;5:363-375.

56 Inoue T, Shingaki R, Sogawa N, Sogawa CA, Asaumi J, Kokeguchi S, Fukui K: Biofilm formation by a fimbriae-deficient mutant of Actinobacillus actinomycetemcomitans. Microbiol Immunol 2003;47:877-881.

57 Kaplan JB: Biofilm dispersal: Mechanisms, clinical implications, and potential therapeutic uses. J Dent Res 2010;89:205-218.

58 Dominiak DM, Nielsen JL, Nielsen PH: Extracellular DNA is abundant and important for microcolony strength in mixed microbial biofilms. Environ Microbiol 2010; doi:10.1111/j.1462-2920.2010.02375.x.

59 Stewart PS, Costerton JW: Antibiotic resistance of bacteria in biofilms. Lancet 2002;358:135-138. 


\section{Figure legends}

Figure 1. Overview of subgingival biofilms taken at a 30-year time interval from extracted tooth. Left images $(21,22)$ are electron microscopic views and the right image is stained with a FITC-labeled universal bacterial probe that stains the bacteria green. Note the remarkable agreement in biofilm structure and observed cell morphologies. C, cementum surface; MC, mammalian cells; $\mathrm{P}$, palisading bacteria; $\mathrm{S}$, spirochete-rich region. The two left images are reproduced from Listgarten [22] with permission from the American Academy of Periodontology.

Figure 2. Overview of the subgingival biofilm with Actinobacteria sp. (green), bacteria (red) and eukaryotic cells (large green cells on top). The four different layers of the biofilm are depicted with the root surface orientated at the bottom of the image. For technical details see reference [23] from which the image was reproduced. Bar $10 \mu \mathrm{m}$.

Figure 3. The intermediate layer of the biofilm is dominated by fusobacteria (yellow, A) and Tannerella forsythia (yellow, B). Modified from [23]. Bars $10 \mu \mathrm{m}$.

Figure 4. Detailed overview of the top layer of the biofilm where Synergistetes sp. (yellow), visible as large perpendicularly orientated cells, are opposing large eukaryotic cells (green), most likely polymorphonuclear leukocytes. Modified from [23]. Bars $10 \mu \mathrm{m}$.

Figure 5. Detail of the outside layer showing the dominance of Treponema sp. (yellow) and the presence of two types of test-tube brush (arrows). Modified from [23].

Figure 6. Localization of typical periodontitis associated species in the biofilm. (A) Overview of the subgingival biofilm with Cytophaga-Flavobacterium-Bacteroides (CFB)-cluster species (red) and Prevotella sp. (yellow). Since Prevotella sp. are part of the CFB-cluster of bacteria, cells appear in yellow. (B) Top of the biofilm with a micro-colony of Parvimonas micra 
(yellow). (C) Micro-colonies of Porphyromonas gingivalis (yellow) in the top layer. (D) Microcolonies of Prevotella endodontalis (yellow) in the top layer. (E) Microcolonies of Prevotella intermedia in the top layer. In panels B - E bacteria are universally stained with a red or green label. Reproduced from [23].

Figure 7. CLSM images of 10-species 'subgingival' in vitro biofilms grown in parallel for 64.5 h on hydroxyapatite (HA) discs in $50 \%$ serum $/ 50 \%$ medium and then fluorescence-labeled by multiplex FISH. Panel A visualizes the 3-D arrangement of transverse (X-Y), sagittal (Y-Z) and coronal (X-Z) CLSM sections shown in the other panels. Panels A and B show sections through the same biofilm stained for Actinomyces naeslundii (red, probe L-Act476-2-Cy3) and all bacteria (green, SYTOX Green/YO-PRO). Black areas are bacteria-free spaces filled by EPS. Bar $20 \mu \mathrm{m}$. BF, biofilm; EPS, extracellular polymeric substances. (C) Biofilm stained for T. denticola (light blue, TrepG1_679-Cy5), P. gingivalis (red, L-Pgin1006-2-C3), all bacteria (green, Sytox and YO-PRO), and extracellular polysaccharides (dark blue, Calcofluor). Note that the two specifically labeled species are located in close proximity to each other near the surface of the biofilm. Bar: $20 \mu \mathrm{m}$. (D) Spatial distribution of $S$. anginosus and S. oralis (both blue, STR405-Cy5), and F. nucleatum (red, Fnuc133c-Cy3) demonstrating their abundance and formation of large self-aggregates. The green-yellow stained bacteria are V. dispar (VEI217-FAM), characteristically located in neighborhood to streptococci and at the top of the biofilm. Bar $30 \mu \mathrm{m}$. Images are from an unpublished study of Ammann et al.. 

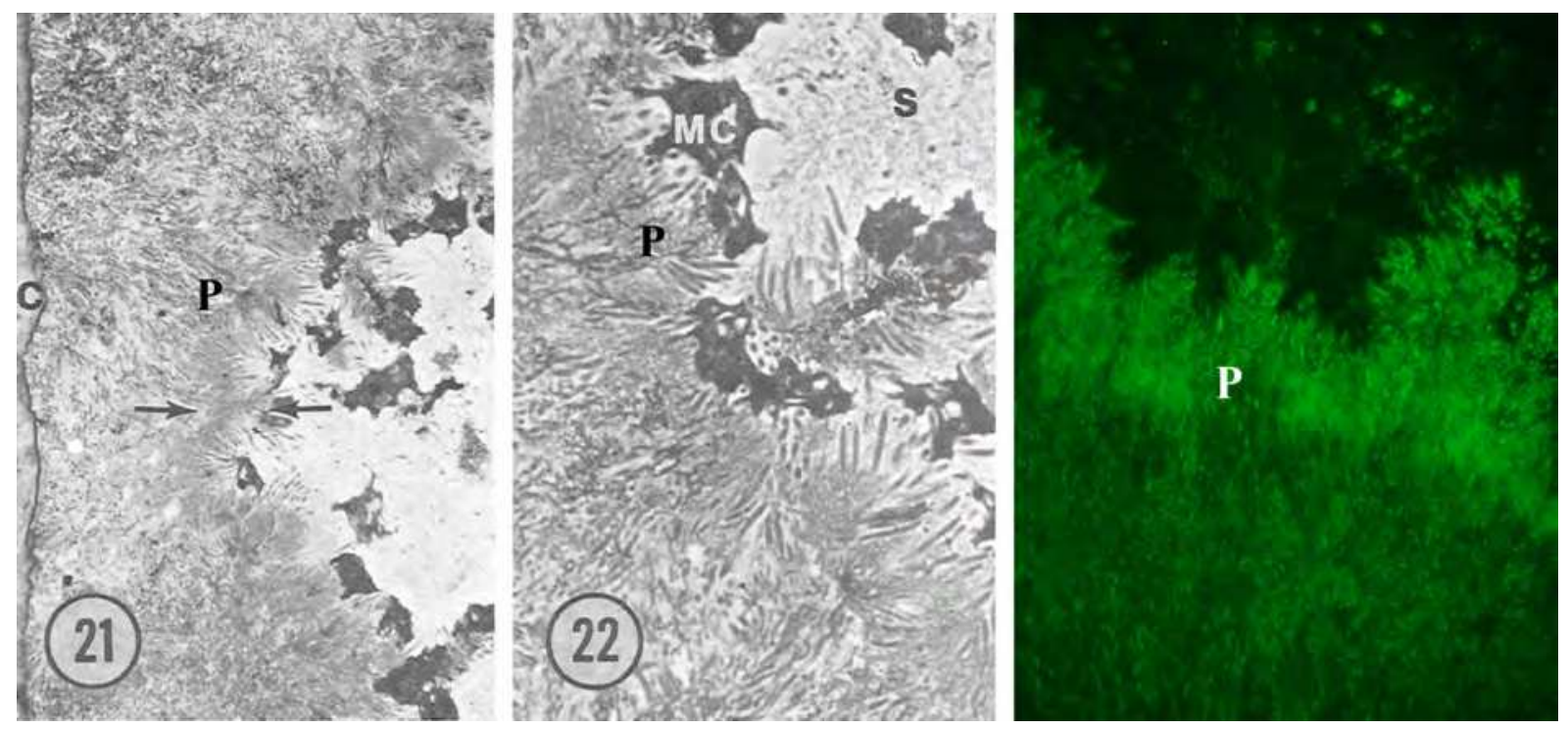

Figure 1

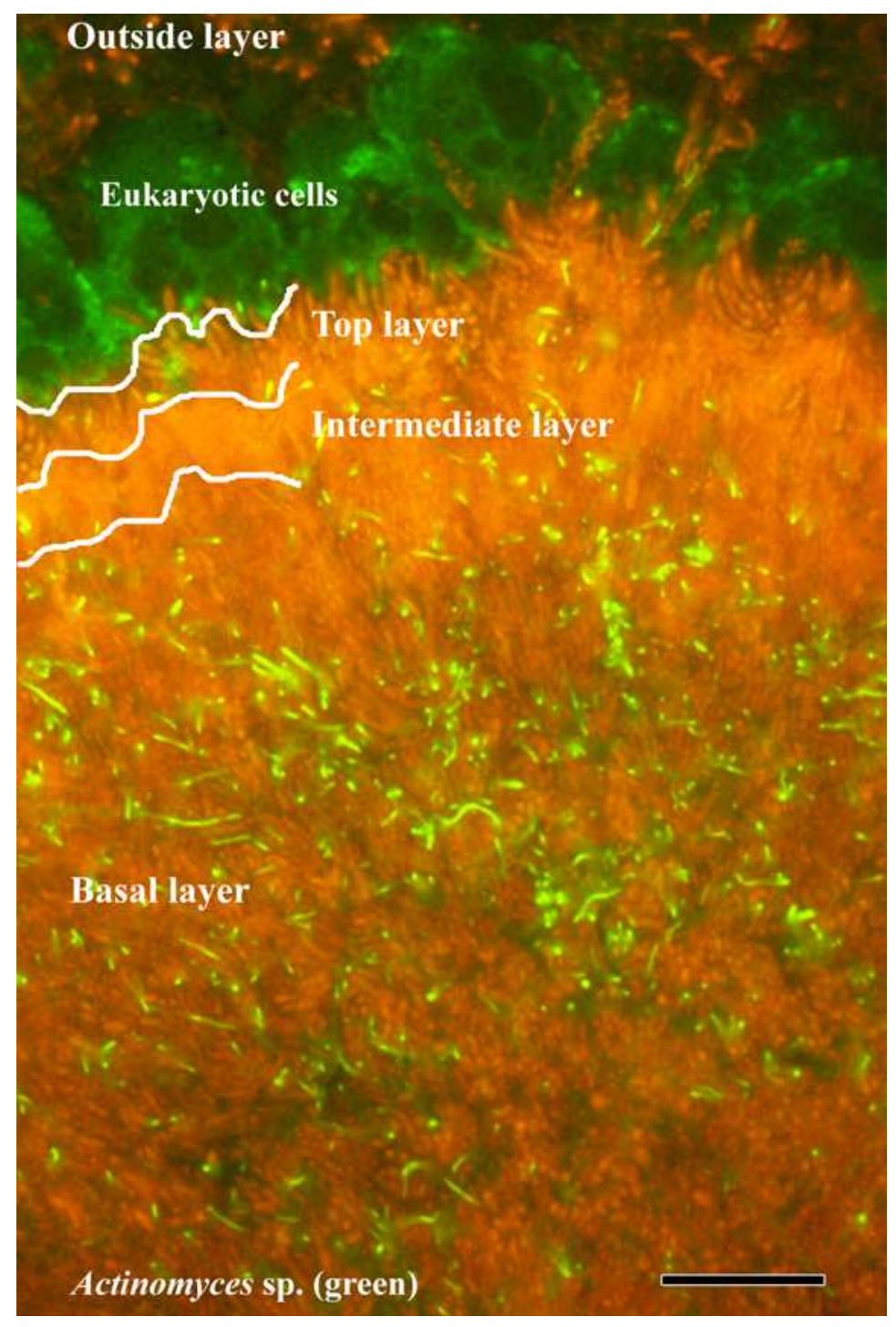

Figure 2 

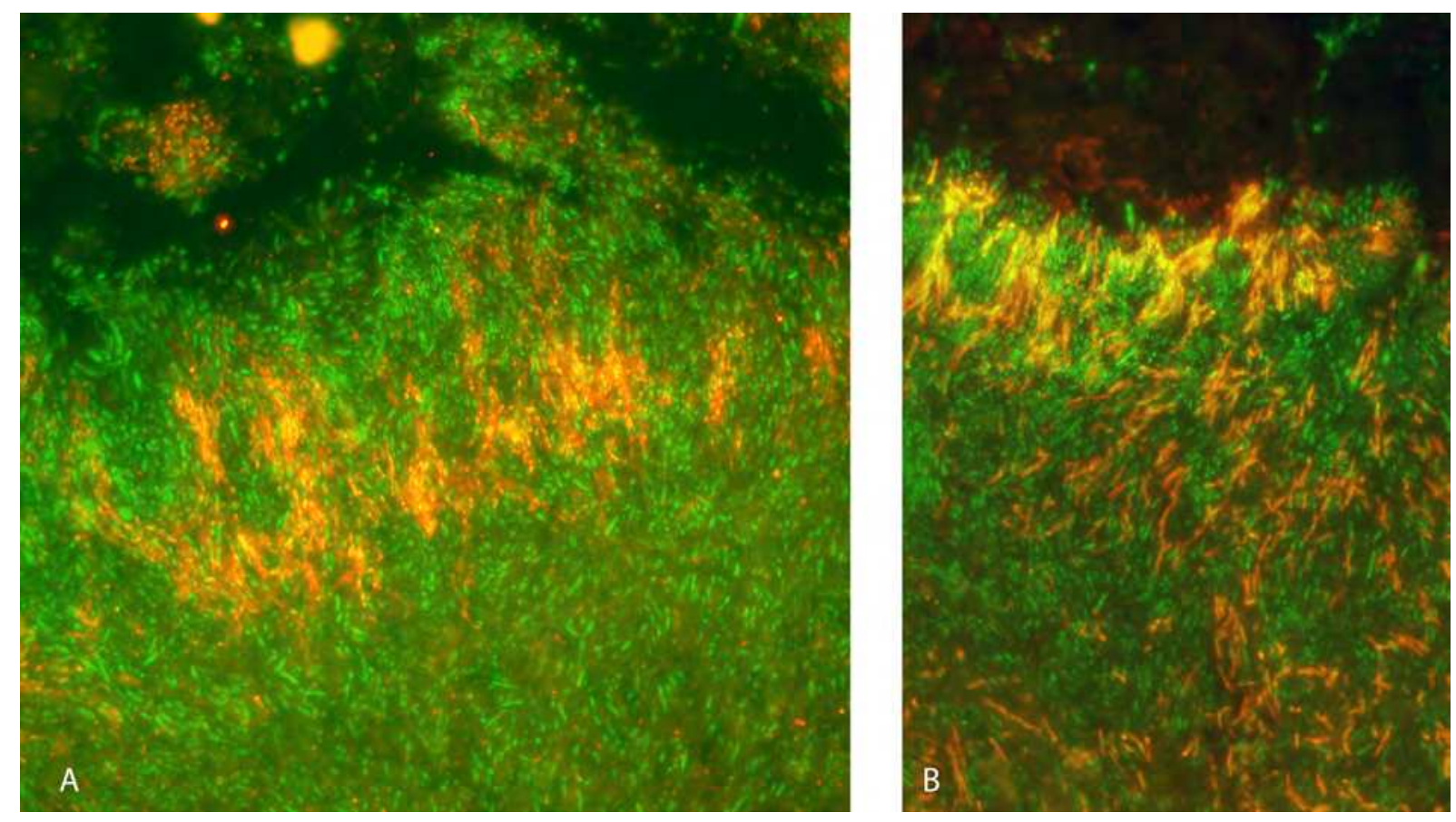

Figure 3

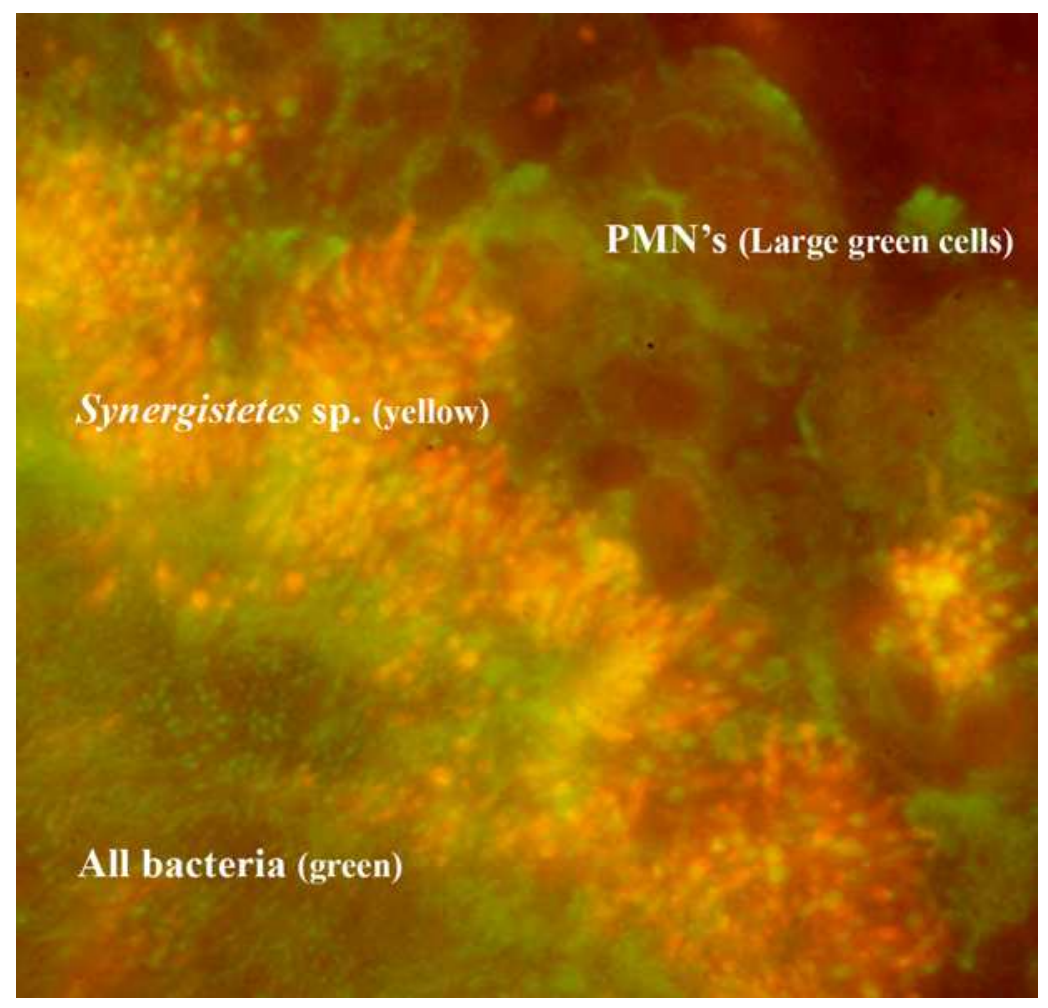

Figure 4 


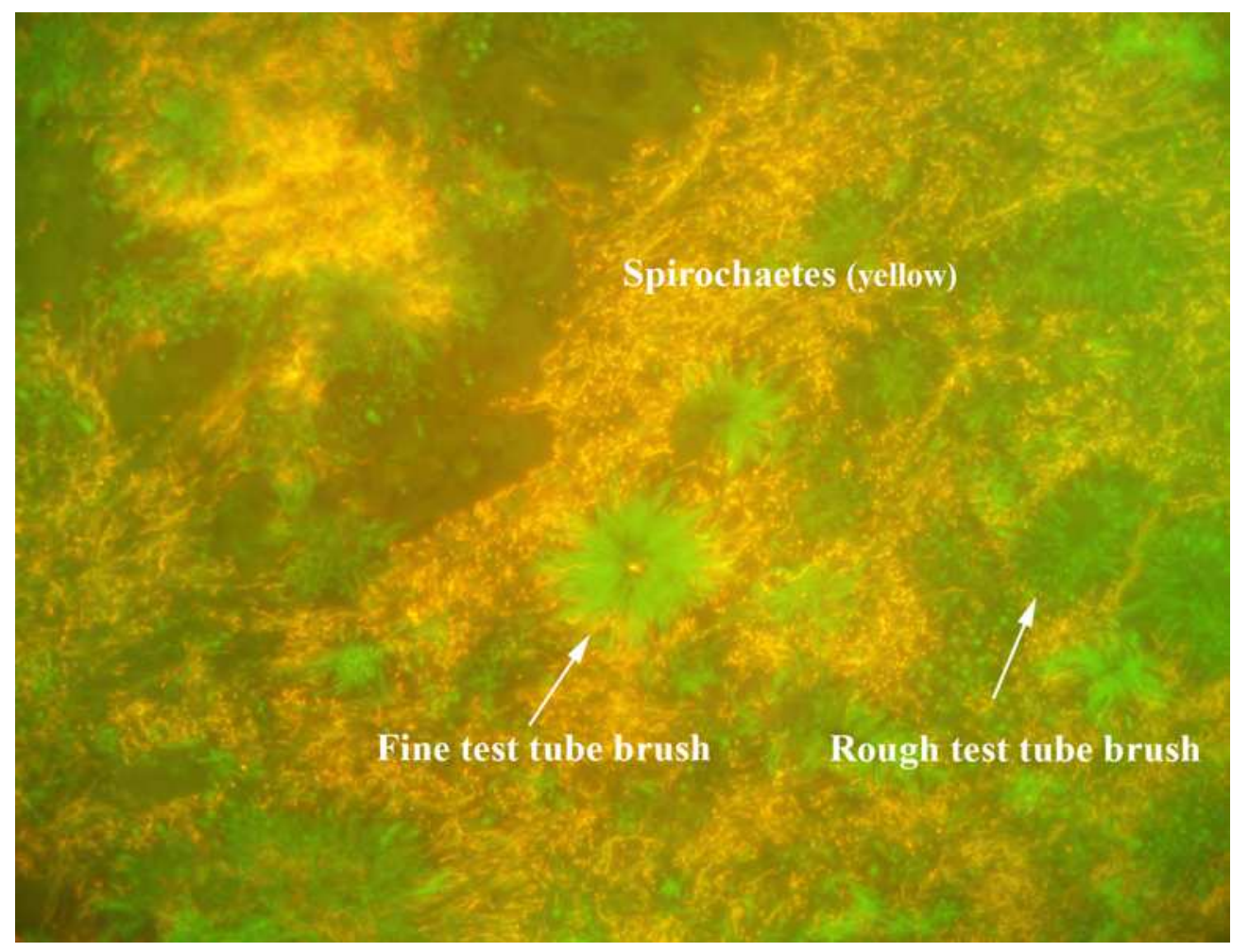

Figure 5
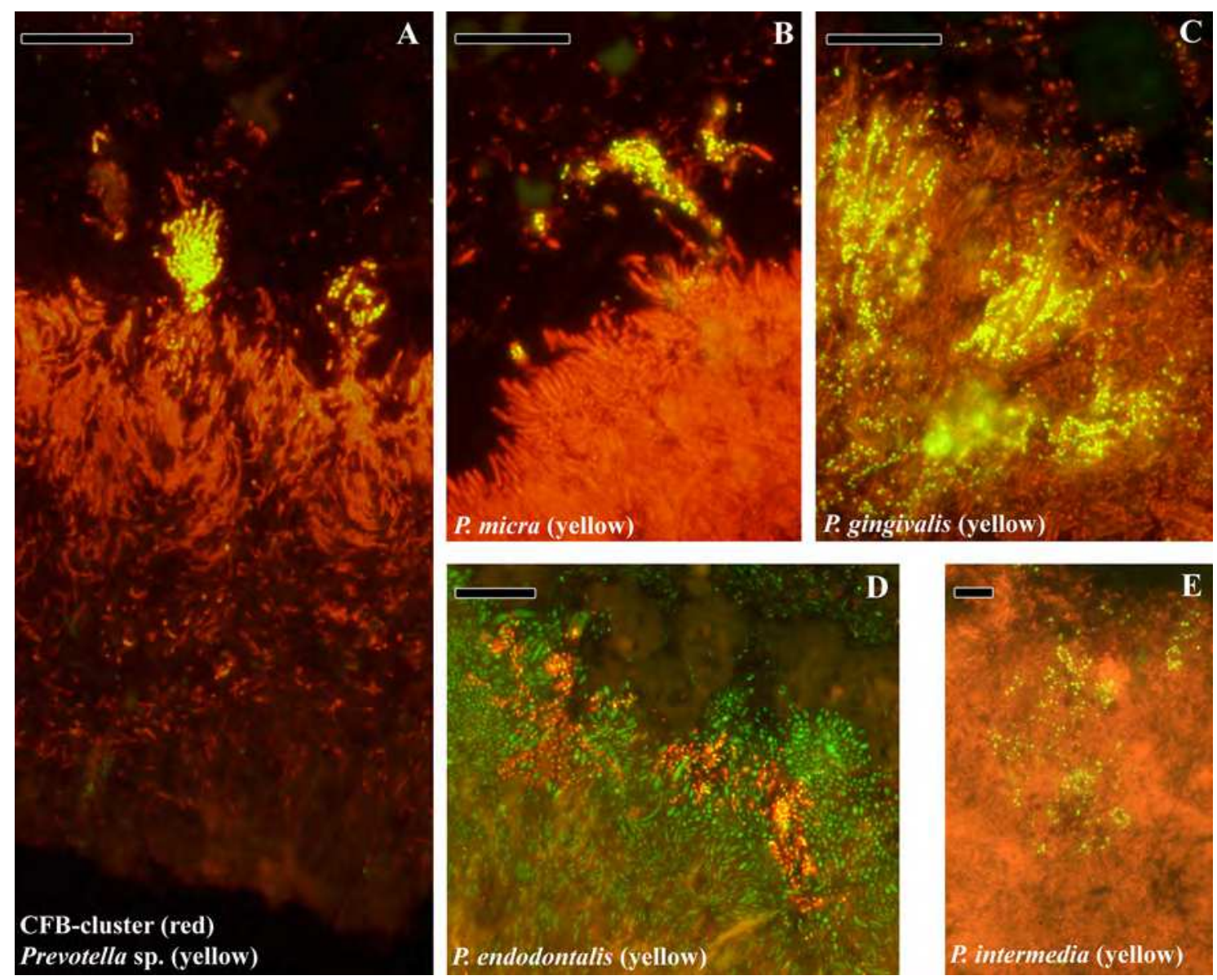

Figure 6 

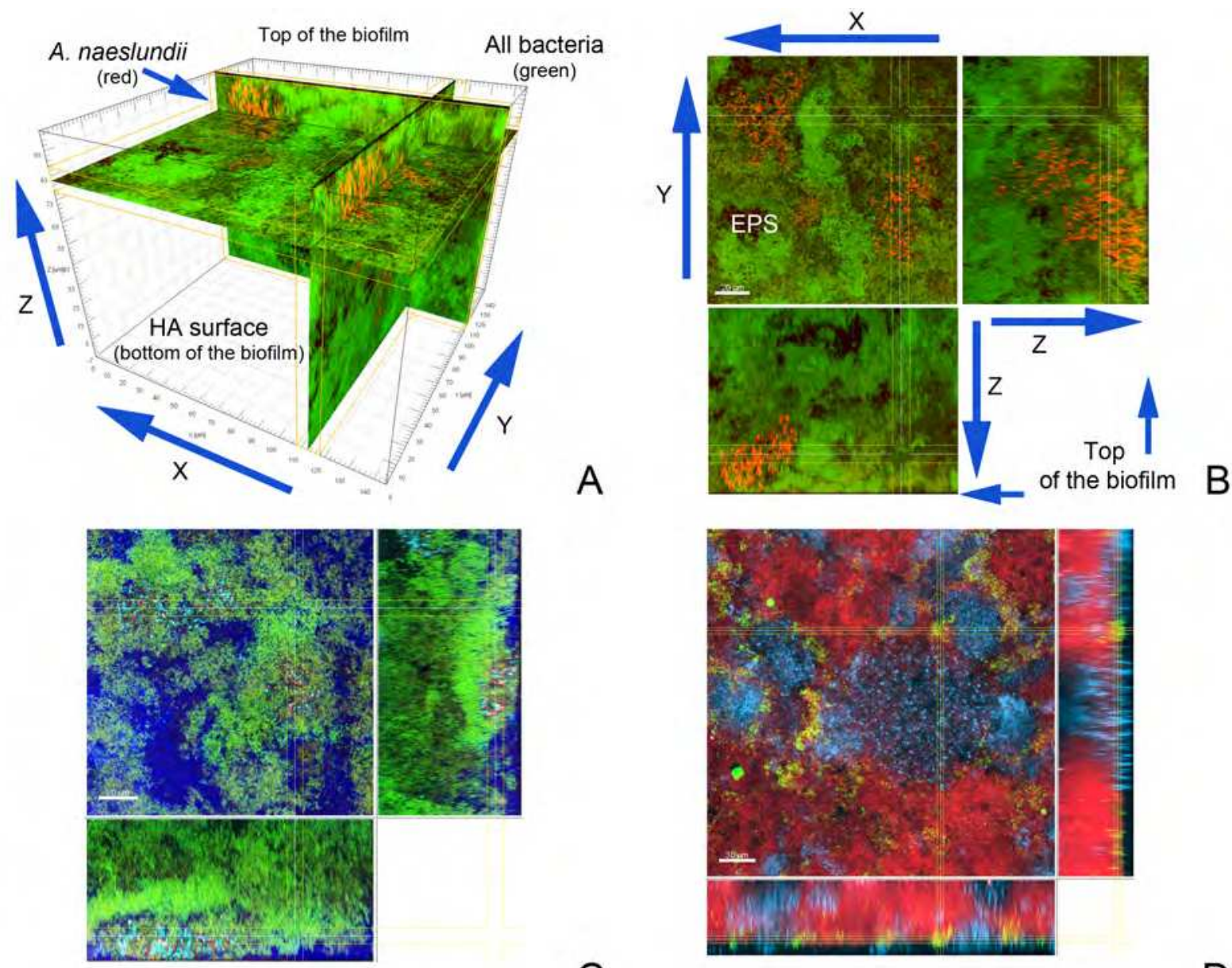

C

D

Figure 7 